\title{
In vivo models of human airway epithelium repair and regeneration
}

\author{
C. Coraux, R. Hajj, P. Lesimple and E. Puchelle
}

ABSTRACT: Despite an efficient defence system, the airway surface epithelium, in permanent contact with the external milieu, is frequently injured by inhaled pollutants, microorganisms and viruses. The response of the airway surface epithelium to an acute injury includes a succession of cellular events varying from the loss of the surface epithelium integrity to partial shedding of the epithelium or even to complete denudation of the basement membrane. The epithelium has then to repair and regenerate to restore its functions. The in vivo study of epithelial regeneration in animal models has shown that airway epithelial cells are able to dedifferentiate, spread, migrate over the denuded basement membrane and progressively redifferentiate to reconstitute a functional respiratory epithelium after several weeks.

Humanised tracheal xenograft models have been developed in immunodeficient nude and severe combined immunodeficient (SCID) mice in order to mimic the natural regeneration process of the human airway epithelium and to analyse the cellular and molecular events involved during the different steps of airway epithelial reconstitution. These models represent very powerful tools for analysing the modulation of the biological functions of the epithelium during its regeneration. They are also very useful for identifying stem/progenitor cells of the human airway epithelium.

A better knowledge of the mechanisms involved in airway epithelium regeneration, as well as the characterisation of the epithelial stem and progenitor cells, may pave the way to regenerative therapeutics, allowing the reconstitution of a functional airway epithelium in numerous respiratory diseases, such as asthma, chronic obstructive pulmonary diseases, cystic fibrosis and bronchiolitis.

KEYWORDS: Airway epithelium, humanised xenografts, nude mice, severe combined immunodeficient mice, stem and progenitor cells

$\mathbf{T}$ he human bronchial airways are lined by a pseudostratified epithelium composed of basal, ciliated and secretory cells, and a subepithelial glandular network responsible for the airway mucus production. The airway epithelial cells are adapted to the defence of the respiratory mucosa by a variety of mechanisms, including mucociliary clearance, secretion of ions, regulation of the airway surface liquid water content, and production of antiinflammatory, antibacterial and antioxidant molecules in the mucus $[1,2]$. The second line of defence is ensured by intercellular junctions that create an efficient barrier against inhaled pathogens or noxious agents intrusion.

Despite this efficient defence system, the airway epithelium is frequently injured because of its permanent contact with the external milieu, which contains toxic agents, viruses, microorganisms and pollutants [3]. Regardless of the source of injury, lesions can vary from the loss of surface epithelium impermeability, as a result of opening of tight junctions, to more or less shedding of the surface airway epithelial cells. After airway epithelial injury, the basement membrane may be completely denuded or partial shedding of the airway surface epithelium may be observed with only clusters of basal cells remaining attached to the basement membrane [4]. These alterations in the epithelial structure and function play a major role in the pathogenesis of numerous respiratory diseases, such as chronic bronchitis, asthma, chronic obstructive pulmonary diseases or cystic fibrosis.

Immediately after injury, the airway epithelium initiates a repair process in order to restore the barrier integrity. The epithelial cells participate, in an orchestrated way, to the closure of the wound and to the functional regeneration of the epithelium. In order to more specifically determine the role of human airway cells and cellular and molecular mechanisms involved in the
AFFILIATIONS

INSERM UMRS 514, IFR 53, CHU

Maison Blanche, Reims, France.

CORRESPONDENCE

E. Puchelle

INSERM UMRS 514

CHU Maison Blanche

45 rue Cognacq Jay

51092 Reims Cedex

France

Fax: 33326065861

E-mail: edith.puchelle@univ-reims.fr 
airway epithelial repair and regeneration, several in vivo models have been described in the literature [5]. This article aims to describe these models.

\section{ANIMAL MODELS OF AIRWAY EPITHELIUM REGENERATION}

A variety of animal models (dogs, rabbits, rats, mice, etc.) have been developed in order to analyse the repair process of the airway epithelium after injury from different sources (oxidants, bacterial or viral infection, mechanical injury, etc.). These animal models highlight a common process in epithelial repair and regeneration, including sequential steps of spreading and migration of the basal cells neighbouring the wound, rapid restoration of the tight junctions, pre-mitosis and dedifferentiation of repairing cells, followed by epithelial squamous metaplasia, active mitosis leading to basal and mucous cell hyperplasia, progressive redifferentiation, with the emergence of pre-ciliated cells (a mixed phenotype of ciliated and mucous cells), and a final step of ciliogenesis representative of the complete regeneration of a mucociliary epithelium [6] (fig. 1).

The regeneration of the hamster tracheal epithelium after mechanical injury has been particularly well described. MCDOWELL et al. [8] reported a rapid re-epithelialisation of the denuded basement membrane with a first step of epithelial migration before all proliferation processes. Indeed, epithelial cells at the wound edge are able to dedifferentiate, spread and then migrate to completely cover the denuded basal lamina.

Animal tracheal xenograft models have also been developed in order to analyse animal airway epithelium repair and regeneration and to identify stem or progenitor cells involved in the airway epithelium reconstitution. Induction of the regeneration process in animal airway tissue, which is normally characterised by a low turnover, accelerates the cell proliferation and differentiation. All these studies suggest that several categories of stem cells and progenitor cells, including surface columnar [9] and basal cells [10], as well as ductal cells of the submucosal glands [11], can participate in airway epithelium regeneration and renewal. In the rat xenograft model, adult rat tracheal epithelial cells are seeded on a denuded rat trachea implanted on the back of a nude mouse. After a few days, dedifferentiated cells, exhibiting markers of basal cells, colonise the basement membrane and then develop the typical features of the rat airway epithelium from which they originate [12]. Rat tracheal secretory cells, isolated by flow cytometry, have a greater colony-forming efficiency than basal cells and could be the airway stem cells [13].

Nevertheless, histological differences exist between human and animal airways, raising doubts as to the relevance of the latter as models for the study of the human airway epithelium regeneration. For example, mouse tracheal epithelium is composed mainly of ciliated cells and Clara cells, the latter being present only in human distal bronchiolar airways, whereas only few submucosal gland cells are identified at the upper tracheal level in mice when they are identified in human upper and lower airways.

\section{HUMAN AIRWAY XENOGRAFT MODELS}

Human airway xenograft models of maturation and regeneration have been developed during the last decade [5]. In order to identify and trace progenitor cells of the human airway epithelium, ENGELHARDT et al. [14] and ZEPEDA et al. [15] adapted the animal xenograft model reported by SHIMIZU et al. [12] in 1992. They seeded dissociated human airway epithelial cells into a host rat trachea denuded from its own epithelium and then grafted into an immunodeficient mouse. By using this model, they could observe the regeneration of a welldifferentiated mucociliary human airway epithelium and demonstrated that submucosal gland progenitor cells were present within the surface airway epithelium, as clonal analysis revealed that more than one airway progenitor cell was involved in the initial stage of gland development, and that several stem cell subsets could be responsible for airway epithelial regeneration [16].

\section{HUMAN ADULT AIRWAY EPITHELIAL REGENERATION IN A NUDE MOUSE XENOGRAFT MODEL}

To analyse the regeneration and maturation process of the human airway epithelium, the present authors adapted the tracheal xenograft model in nude mice [17], initially developed in 1992 by ENGELHARDT et al. [14]. Human airway epithelial cells either dissociated from nasal polyps or bronchi were seeded into the lumen of rat tracheas denuded from their own epithelium by successive cycles of freezing and thawing. The host tracheas were tied to sterile polyethylene tubing at their

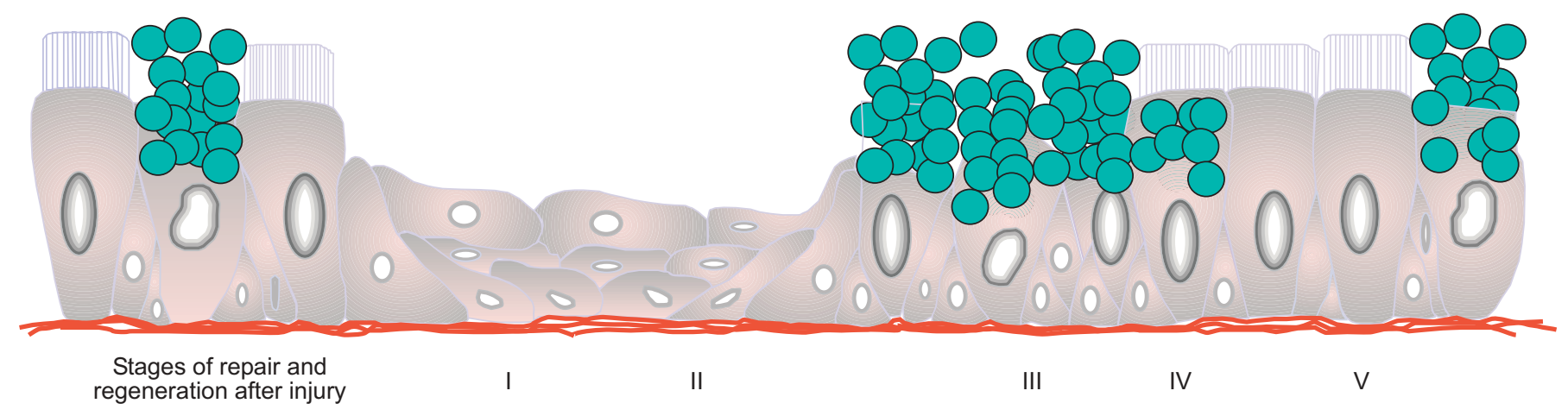

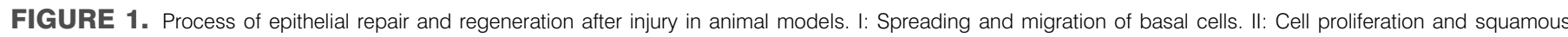

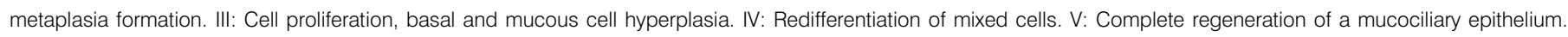
Reproduced with modification from [7] with permission from the publisher. 
distal ends and implanted subcutaneously in the flanks of recipient nude mice. This "air-opened" chimeric model has the advantage of allowing the reconstitution of a human airway epithelium exposed to the external milieu in the same way as in adult human airways [18] (fig. 2). The sequence of cellular events leading to the complete regeneration of the human airway epithelium was determined; this is similar to the human epithelium regeneration described after airway injury. After 3 days of engraftment, tracheas were partly repopulated with a flattened, nonciliated and poorly differentiated untight epithelium. After 13 days, cell proliferation occurred and led to the formation of a squamous-type epithelium that was stratified into multiple layers and tightly sealed, and covered the entire surface of the host rat tracheas. This squamous epithelium phenotype, which represents a highly protective phenotype previously described after injury, does in fact reflect a protective dynamic regenerative process. At 25 days after implantation, the cell proliferation was stopped and the epithelium became progressively pseudostratified, with ciliated and secretory cell differentiation; it was still partly untight, although it was already polarised. After 35 days of engraftment, the epithelium was fully differentiated and tight, exhibited the cystic fibrosis transmembrane conductance regulator (CFTR) chloride channel at the apical domain of the ciliated cells and submucosal glandular structures were visible.
Recently, the "air-opened" humanised xenograft model, which mimics the regeneration dynamics after severe injury, was used to characterise the cellular and molecular mechanisms involved during the different steps of airway epithelial reconstitution. The expression and potential involvement of some matrix metalloproteinases (MMPs), matrilysin (MMP-7) and gelatinase B (MMP-9), and of their inhibitor, the tissue inhibitor of matrix metalloproteinases-1 (TIMP-1), were analysed, as well as of the pro-inflammatory cytokine interleukin (IL)-8. It was found that during the cell migration and proliferation steps, airway epithelial cells expressed IL-8 at a high level, whereas airway epithelial pseudostratification and surface airway epithelium differentiation were associated with an increased expression of MMPs and a progressive decrease of IL-8. Interestingly, the immunohistochemical detection revealed an exclusive expression of MMPs at the apical part of the well-differentiated regenerated airway epithelium, and incubation of the regenerating epithelial cells with MMP inhibitors led to an abnormal epithelial differentiation attested by immature and mature squamous metaplasia with areas of basal cell hyperplasia. These data provide a new insight into the temporal expression of MMPs and IL- 8 during the human airway epithelium regeneration, and demonstrate the involvement of these factors during the different steps, leading to the restoration of a well-differentiated and functional airway epithelium [19] (fig. 3).

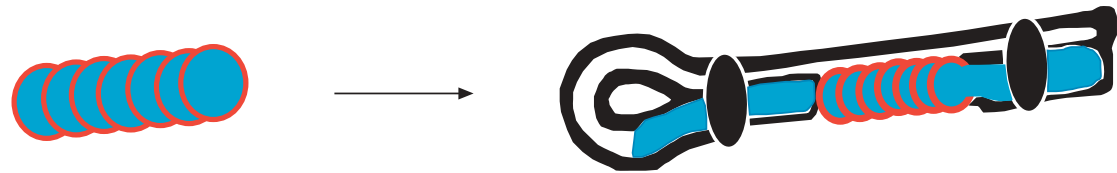

Denuded rat trachea

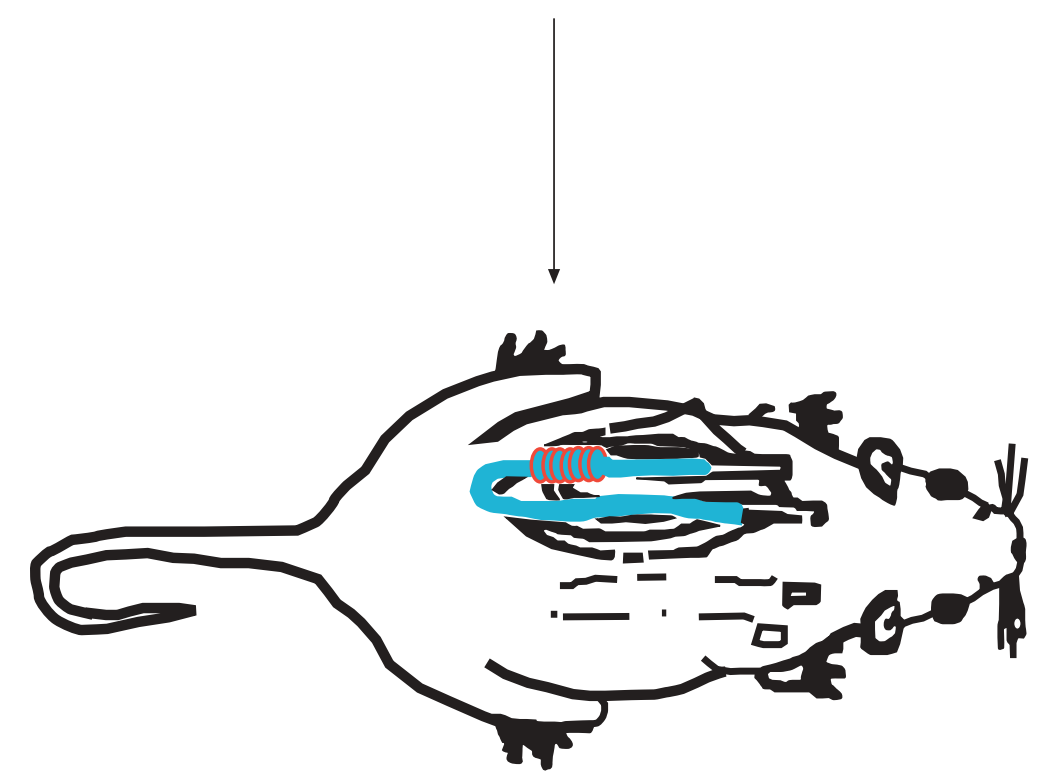

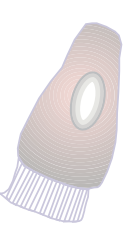

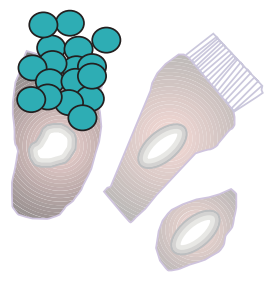

Human airway epithelial cells

Subcutaneous implantation in a nude mouse

FIGURE 2. Air-opened nude mouse xenograft model. Dissociated airway epithelial cells are seeded into the lumen of a denuded rat trachea tied at their end to sterile tubing. The assembly is subcutaneously implanted in the flank of a recipient nude mouse. Reproduced with modification from [14] with permission from the publisher. 

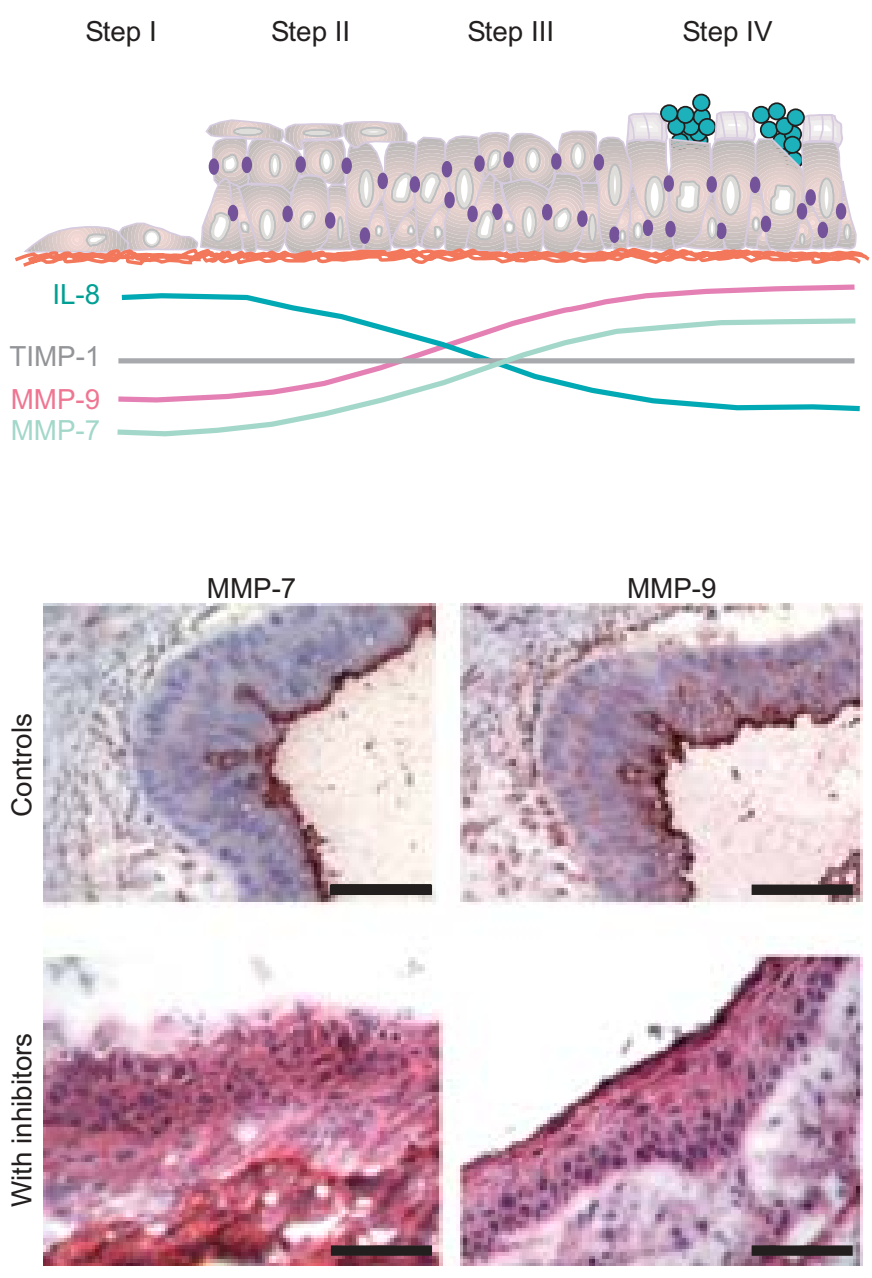

FIGURE 3. Involvement of matrix metalloproteinases (MMPs) and interleukin (IL)-8 during human airway epithelial regeneration in the nude mouse xenograft model. During the different steps of human airway epithelium regeneration, a progressive decrease in IL-8 expression and secretion, associated with a progressive increase in MMPs' expression and secretion, can be observed. When the airway epithelium is fully differentiated, MMPs exhibit an exclusive expression at the apical part of the epithelium, and incubation with MMP inhibitors leads to an abnormal epithelial differentiation attested by immature and mature squamous metaplasia with areas of basal cell hyperplasia. TIMP: tissue inhibitor of matrix metalloproteinase. Scale bars $=35 \mu \mathrm{m}$. Reproduced with modification from [19] with permission from the publisher.

In terms of future human airway cell/gene therapy perspectives, the humanised xenograft model in nude mouse has also been modified and miniaturised to generate a pre-clinical model of cell repopulation assay. CASTILLON et al. [20] cultured human airway epithelial cells as three-dimensional structures called spheroids, allowing epithelial cells to differentiate and to be maintained in a differentiated stage for several months. They then seeded these epithelial three-dimensional differentiated structures on mouse tracheas denuded from their own epithelium, followed by a subcutaneous implantation in nude mice. The authors reported that the spheroids, which can be transduced by a lentiviral vector with sustained expression of the transgene eGFP for $\geqslant 80$ days, were able to adhere and to repopulate the denuded basement membrane after 1-2 weeks of engraftment and, after 4 weeks, to reconstitute a fully differentiated airway epithelium composed of basal, ciliated and secretory cells, exhibiting tight junctions and the CFTR chloride channel at the apical domain of the ciliated cells [21] (fig. 4).

\section{HUMAN FOETAL AIRWAY EPITHELIAL XENOGRAFTS IN SEVERE COMBINED IMMUNODEFICIENT MICE}

Another model of in vivo human airway development using transplantation of embryonic and foetal human lung rudiments into severe combined immunodeficient (SCID) mice has been reported. In this model, proximal airway primordia grew rapidly and differentiated after 6-12 weeks into tracheal structures, including a pseudostratified mucociliary surface epithelium with submucosal gland network. Whatever the initial stage of development, fragments of human foetal tracheas implanted subcutaneously into SCID mice were rapidly closed at each end by a neoformed membrane (called operculum) that lined their whole inner surface with a pseudostratified and secretory epithelium. The presence of airway fluid inside the lumen of these tracheal grafts resulted from surface cell and submucosal gland secretion [22].

This humanised SCID mice xenograft model was recently used as an assay for airway progenitor cells. For this purpose, tracheal grafts were first repopulated with allogeneic epithelial cells: total epithelial cells dissociated from developed airways were seeded in host grafts from which native epithelium had been eliminated by successive rounds of freezing/thawing. The recovery of a fully differentiated mucociliary epithelium ensued in all cases, whereas autonomous regeneration did not occur in control unseeded grafts. The donor origin of newly formed epithelia was confirmed in sex-mismatched combinations of hosts and donor tissues. In other experiments, the endodermal pouch dissociated from 5-7 week embryonic lung rudiment, which forms a homogenous population of respiratory epithelial stem cells, was used as donor tissue. Such early anlagen also replenished the denuded host grafts with the full spectrum of surface epithelial cells and glands. These experimental results demonstrate that candidate airway epithelial stem cells can be assayed functionally in this model [23]. The search for molecular markers discriminating such progenitors is being actively pursued. Indeed, the same group screened an array of candidate markers, among them the aquaporin-3 (AQP3) water channel, specifically expressed on the surface of human foetal tracheal basal cells and allowing the separation by flow cytometry of AQP3+ foetal basal cells and AQP3- foetal ciliated and secretory cells. Functional evaluation of sorted cells in the humanised SCID mice xenograft model showed that AQP3+ cells restored a normal pseudostratified mucociliary epithelium, as well as submucosal glands. AQP3- cells also exhibited the same potential but with faster engraftment, suggesting their inclusion of more committed progenitors. These results showed that epithelial progenitors exist among both basal and suprabasal cell subsets within human foetal airways [24].

\section{CONCLUSIONS}

The regeneration process of the human airway epithelium is a complex phenomenon, partly elucidated by the use of animal models. However, some histological differences between human airways and those of other animal species led to the 

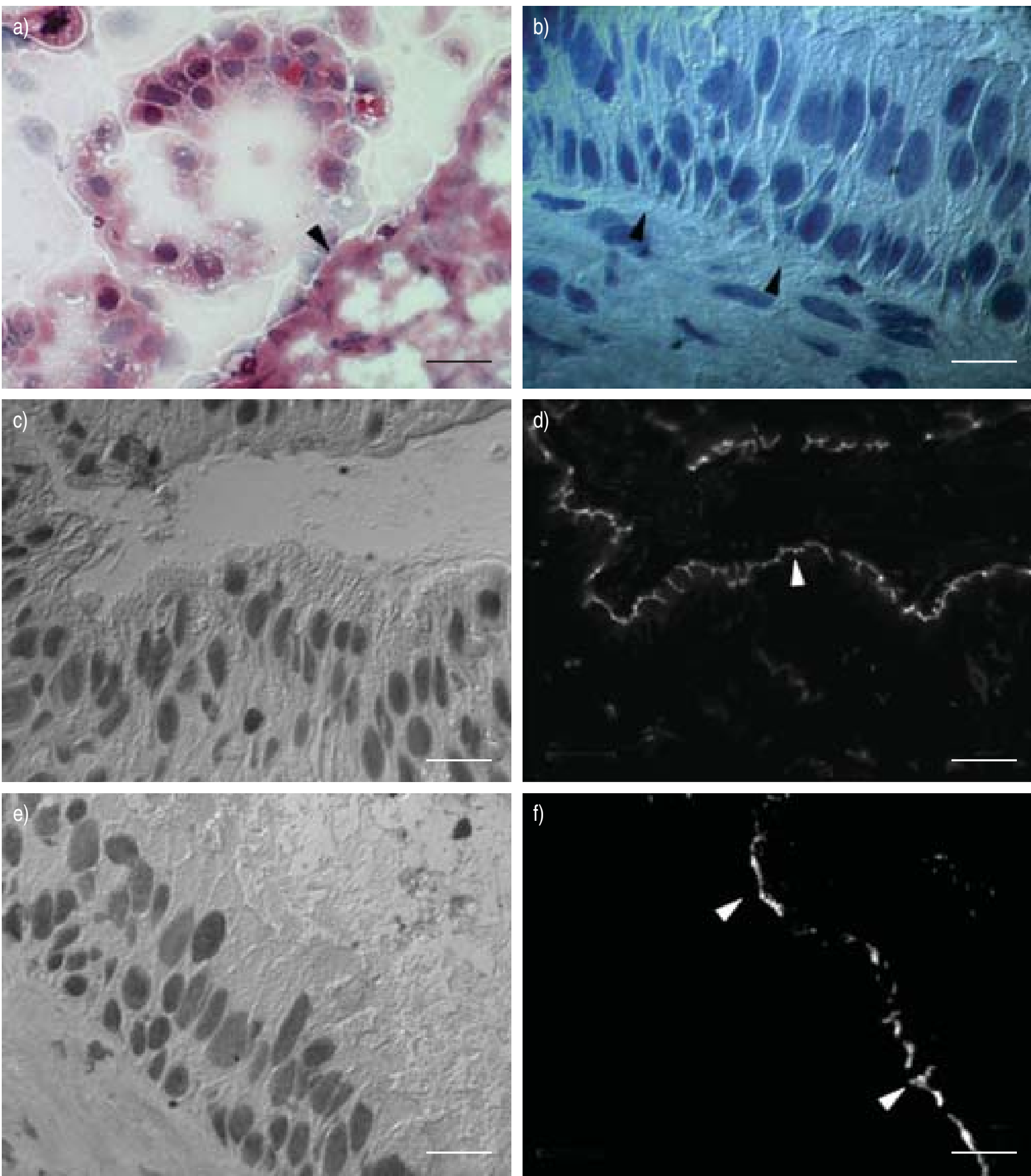

FIGURE 4. Regeneration of human airway epithelium in a miniaturised nude mouse xenograft model using airway epithelial cells cultured as three-dimensional spheroids. a) After 1-2 weeks of engraftment, spheroids have adhered to the denuded basement membrane. After 4 weeks, airway epithelial cells from the spheroids were able to reconstitute a fully differentiated airway epithelium (b) exhibiting tight junctions, attested by the immunostaining of the ZO-1 protein (c and d), and the cystic fibrosis transmembrane conductance regulator chloride channel at the apical domain of the ciliated cells (e and f). Scale bars $=20 \mu \mathrm{m}$ (a) and $10 \mu \mathrm{m}$ (b-f). Reproduced with modification from [21] with permission from the publisher. 
development of humanised xenograft models in immunodeficient mice in order to characterise the cellular and molecular events involved in the reconstitution of a fully mature and functional human airway epithelium, as well as to try to identify the stem and progenitor cells implicated in this process. These xenograft models will allow a better knowledge of the mechanisms involved in airway epithelium regeneration. They will permit identification of the changes in the defence capacity of the airway epithelium (antibacterial, antiprotease and antioxidant proteins) during the regeneration process and may help to develop regenerative therapeutics allowing the reconstitution of a functional airway epithelium in numerous respiratory diseases, such as asthma, chronic obstructive pulmonary diseases, cystic fibrosis and bronchiolitis.

\section{REFERENCES}

1 Puchelle E, de Bentzmann S, Jacquot J, Zahm JM. Defense properties of airway surface liquid. In: Gibson GJ, Gedded DM Costabel U, Sterk P, Corrin B, eds. Respiratory Medicine. Philadelphia, PA, USA, Saunders, 2003; pp. 194-204.

2 Bals R, Weiner DJ, Wilson JM. The innate immune system in cystic fibrosis lung disease. J Clin Invest 1999; 103: 303-307.

3 Puchelle E, Zahm JM, Tournier JM, de Bentzmann S. Airway epithelial injury and repair. Eur Respir Res 1997; 43: 136-141.

4 Man SFP, Hulbert WC. Airway repair and adaptation to inhalation injury. In: Loke J, ed. Pathophysiology and Treatment of Inhalation Injuries. Lung Biology in Health and Disease. New York, NY, USA, Dekker, 1988; pp. 1-47.

5 Puchelle E, Peault B. Human airway xenograft models of epithelial cell regeneration. Respir Res 2000; 1: 125-128.

6 Puchelle E, Zahm JM. Repair process of the airway epithelium. In: Chretien J, Dusser D, eds. Environmental Impact on the Airways. From Injury to Repair. New York, NY, USA, Dekker, 1996; pp. 157-182.

7 Puchelle E. Airway epithelium wound repair and regeneration after injury. Acta Otorhinolaryngol Belg 2000; 54: 263-270.

8 McDowell EM, Becci PJ, Schurch W, Trump BF. The respiratory epithelium. VII. Epidermoid metaplasia of hamster tracheal epithelium during regeneration following mechanical injury. J Natl Cancer Inst 1979; 62: 995-1008.

9 Hong KU, Reynolds SD, Giangreco A, Hurley CM, Stripp BR. Clara cell secretory protein-expressing cells of the airway neuroepithelial body microenvironment include a label-retaining subset and are critical for epithelial renewal after progenitor cell depletion. Am J Respir Cell Mol Biol 2001; 24: 671-681.

10 Hong KU, Reynolds SD, Watkins S, Fuchs E, Stripp BR. Basal cells are a multipotent progenitor capable of renewing the bronchial epithelium. Am J Pathol 2004; 164: 577-588.
11 Borthwick DW, Shahbazian M, Todd Krantz Q, Dorin J, Randell SH. Evidence for stem-cell niches in the tracheal epithelium. Am J Respir Cell Mol Biol 2001; 24: 662-670.

12 Shimizu T, Nettesheim P, Ramaekers FC, Randell SH. Expression of "cell-type-specific" markers during rat tracheal epithelial regeneration. Am J Respir Cell Mol Biol 1992; 7: 30-41.

13 Liu JY, Nettesheim P, Randell SH. Growth and differentiation of tracheal epithelial progenitor cells. Am J Physiol 1994; 266: L296-L307.

14 Engelhardt JF, Yankaskas JR, Wilson JM. In vivo retroviral gene transfer into human bronchial epithelia of xenografts. J Clin Invest 1992; 90: 2598-2607.

15 Zepeda ML, Chinoy MR, Wilson JM. Characterization of stem cells in human airway capable of reconstituting a fully differentiated bronchial epithelium. Somat Cell Mol Genet 1995; 21: 61-73.

16 Engelhardt JF, Schlossberg H, Yankaskas JR, Dudus L. Progenitor cells of the adult human airway involved in submucosal gland development. Development 1995; 121: 2031-2046.

17 Dupuit F, Gaillard D, Hinnrasky J, et al. Differentiated and functional human airway epithelium regeneration in tracheal xenografts. Am J Physiol Lung Cell Mol Physiol 2000; 278: L165-L176.

18 Escotte S, Catusse C, Coraux C, Puchelle E. Reconstitution of human airway tissue in the humanized xenograft model. J Cyst Fibros 2004; 3: Suppl. 2, 63-65.

19 Coraux C, Martinella-Catusse C, Nawrocki-Raby B, et al. Differential expression of matrix metalloproteinases and interleukin-8 during regeneration of human airway epithelium in vivo. J Pathol 2005; 206: 160-169.

20 Castillon N, Hinnrasky J, Zahm JM, et al. Polarized expression of cystic fibrosis transmembrane conductance regulator and associated epithelial proteins during the regeneration of human airway surface epithelium in threedimensional culture. Lab Invest 2002; 82: 989-998.

21 Castillon N, Avril-Delplanque A, Coraux C, et al. Regeneration of a well-differentiated human airway surface epithelium by spheroid and lentivirus vectortransduced airway cells. J Gene Med 2004; 6: 846-856.

22 Peault B, Tirouvanziam R, Sombardier MN, Chen S, Perricaudet M, Gaillard D. Gene transfer to human fetal pulmonary tissue developed in immunodeficient SCID mice. Hum Gene Ther 1994; 5: 1131-1137.

23 Delplanque A, Coraux C, Tirouvanziam R, et al. Epithelial stem cell-mediated development of the human respiratory mucosa in SCID mice. J Cell Sci 2000; 113: 767-778.

24 Avril-Delplanque A, Casal I, Castillon N, Hinnrasky J, Puchelle E, Peault B. Aquaporin-3 expression in human fetal airway epithelial progenitor cells. Stem Cells 2005; 23: 992-1001. 